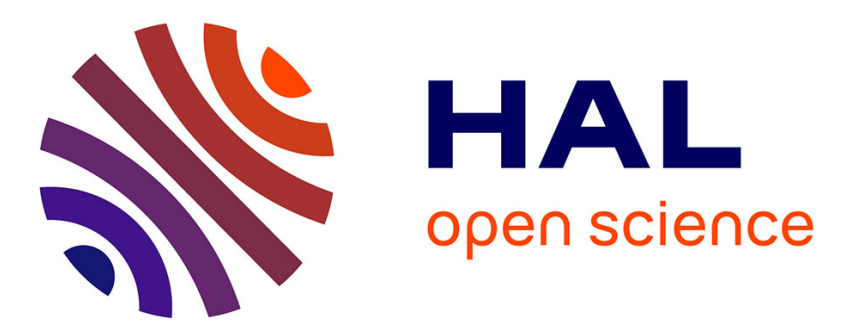

\title{
Influence d'un complément multivitaminé comprenant du Guarana sur la réponse cardiovasculaire et la performance cognitive
}

\author{
Philippe Le Van, Jeanick Brisswalter
}

\section{- To cite this version:}

Philippe Le Van, Jeanick Brisswalter. Influence d'un complément multivitaminé comprenant du Guarana sur la réponse cardiovasculaire et la performance cognitive. Movement \& Sport Sciences - Science \& Motricité, 2012, 76, pp.39-46. hal-01727383

\section{HAL Id: hal-01727383 https://hal-insep.archives-ouvertes.fr/hal-01727383}

Submitted on 9 Mar 2018

HAL is a multi-disciplinary open access archive for the deposit and dissemination of scientific research documents, whether they are published or not. The documents may come from teaching and research institutions in France or abroad, or from public or private research centers.
L'archive ouverte pluridisciplinaire HAL, est destinée au dépôt et à la diffusion de documents scientifiques de niveau recherche, publiés ou non, émanant des établissements d'enseignement et de recherche français ou étrangers, des laboratoires publics ou privés. 


\title{
Influence d'un complément multivitaminé comprenant du Guarana sur la réponse cardiovasculaire et la performance cognitive
}

\author{
Philippe Le $\operatorname{Van}^{1}$ et Jeanick Brisswalter ${ }^{2}$ \\ ${ }^{1}$ Département Médical, Institut National du Sport de l'Expertise et de la Performance, INSEP, 11 avenue du Tremblay, \\ 75012 Paris, France \\ ${ }^{2}$ Laboratoire d'Analyse de la Motricité Humaine Éducation Santé LAMHES, Université de Nice Sophia Antipolis, BP \\ 3259, 06205 Nice, France
}

Article publié dans : Movement \& sport sciences - Science \& motricité (ISSN 2118-5735), 2012, $n^{\circ} 76$, pp. $39-46$

\section{Résumé.}

L'objectif de cette étude était de comparer l'effet, d'un complément multivitaminé avec du Guarana, de caféine, ou d'un placebo, sur la performance cognitive et la réponse cardiovasculaire. 56 sujets ont participé à 4 sessions expérimentales. Des tâches perceptuelles, décisionnelles et de vigilance ont été présentées pendant $3 \mathrm{~h}$ après l'ingestion du complément. La fréquence cardiaque et la pression artérielle étaient mesurées. Les résultats indiquent un effet positif du complément et de la caféine sur la performance décisionnelle et la vigilance après $30 \mathrm{~min}$. Cet effet est plus important entre 30 et $120 \mathrm{~min}$ pour le complément que pour la caféine. Aucun effet n'est observé sur la réponse cardiovasculaire. Ces résultats posent la question de l'intérêt de ce type de complément pour le sportif.

Mots clés : Vitamines, Guarana, tâche décisionnelle, attention, cognition

\begin{abstract}
.
Effects of a multi-vitamin supplement with added Guarana on cardiovascular response and cognitive performance.

The aim of this study was to compare the effects of a multi vitamin and Guarana supplement, caffeine, or a placebo, on cognitive performance and cardiovascular responses. 56 subjects participated in four experimental sessions. Perceptual, decisional and vigilance tasks were presented during a 3 hour period after ingestion. Heart rate and blood pressure were continuously recorded. Results indicate a positive effect of the supplement and caffeine on decisional and vigilance performance after 30 min. Furthermore this effect was higher between 30 and 120 min with the supplement when compared with caffeine. No effect was observed on cardiovascular response. These results raised the question of the interest of this supplement in a sport context.
\end{abstract}

Key words: Vitamins, Guarana, decisional task, attention, cognition

\section{Introduction}

Dans les activités physiques et sportives, la performance présente un caractère multi-déterminé. Elle dépend à la fois de l'efficacité des processus cognitifs et physiologiques sollicités dans l'action (e.g. Brisswalter et al., 2002). Aussi, de nombreuses études scientifiques ont analysé l'effet de différentes stratégies d'intervention pour optimiser le rendement cognitif, et ceci notamment dans le domaine militaire ou sportif. Parmi ces stratégies, l'effet positif d'un apport de caféine sur la performance cognitive en dehors ou pendant un exercice physique a été largement étudié dans le domaine militaire (e.g. Fredholm et al., 1999 ; Lieberman, 2003 ; McLellan et al., 2007), mais aussi dans le domaine sportif montrant un effet significatif de l'ingestion de caféine sur la performance sportive globale 
(Foskett \& Gant, 2009; Rogers \& Dinges, 2005). Les principaux résultats de ces études réalisées avec ou sans privation de sommeil, ont montré des effets bénéfiques de la caféine sur la vigilance et sur l'éveil en général, sans altération de la précision du geste (e.g. Johnson \& Merullo, 2000). La caféine traverse facilement la barrière hémato-encéphalique et semble exercer des effets sur le cerveau en modulant les récepteurs d'adénosine qui participent à la régulation de l'éveil cortical (Lieberman, 2003). Lorsque la caféine est consommée une heure avant les tests, il a été montré que des doses de 100 à $300 \mathrm{mg}$ ont un effet positif principalement sur la vigilance visuelle alors que l'effet semble moindre pour les tâches cognitives décisionnelles complexes avec un optimum à partir de doses de 200 mg (Lieberman et al., 2002). Néanmoins, dans ces études, souvent menées dans le contexte militaire, les dosages de caféine sont supérieurs à ceux trouvés dans les aliments courants ou compléments alimentaires disponibles pour le sportif. Dans ce cadre, des études récentes ont montré que des composants contenant de la caféine à des doses plus faibles semblent également avoir un effet positif sur la performance cognitive. Parmi celles-ci, quelques études ont montré un effet positif du Guarana qui contient de $5 \%$ à $10 \%$ de caféine sur des tâches de vitesse de mémorisation et la fatigue mentale (Haskell et al., 2007; Kennedy et al., 2008). Cet effet positif est attribué selon les auteurs, à la présence de caféine mais également à d'autres substances chimiques psycho actives contenues dans le Guarana (e.g. tanins) et au fait que le Guarana, dans des complexes alimentaires, est associé à des vitamines et des minéraux.

Par ailleurs malgré ces effets positifs, quelques effets négatifs liés à la présence de caféine doivent être également considéré, incluant par exemple des perturbations de l'homéostasie cardiovasculaire décrites avec l'ingestion de caféine (Clauson et al., 2003).

Dans la littérature analysant les interactions entre les modifications physiologiques ou neurophysiologiques et la cognition, il est clairement montré que ces effets dépendent des processus cognitifs impliqués (perceptifs vs. décisionnels) ou des caractéristiques des sujets, et principalement de leur condition physique (e.g. Brisswalter et al., 2002 ; Lambourne Tomporowski, 2010).

Aussi le but de notre travail est d'observer chez des sujets adultes en bonne condition physique l'influence de l'ingestion d'un supplément à base de Guarana et vitamines sur la réponse cardiovasculaire et les processus cognitifs perceptifs et décisionnels classiquement étudiés dans le domaine sportif. A la suite des travaux de Kennedy et al., 2008, nous émettons l'hypothèse selon laquelle l'ingestion d'un complexe à base de Guarana améliore la performance cognitive de façon plus importante que l'ingestion de caféine seule.

\section{Méthodes et mesures}

\subsection{Sujets}

56 sujets âgés de 29,5 \pm 8 ans ( 32 hommes et 24 femmes) ont participé à ce travail. Les sujets suivaient une pratique sportive régulière (minimum trois séances par semaine). Les sujets ont été sélectionnés pour être exempts d'une pathologie déclarée ou un traitement médical pouvant affecter le niveau de vigilance. Tous les participants étaient des consommateurs habituels de caféine et il leur a été demandé de stopper toute ingestion d'alcool ou de caféine 12 heures avant chaque session de mesure.

\subsection{Plan expérimental}

Le protocole était composé pour chaque sujet de quatre sessions. La première session était toujours une session d'apprentissage des tâches cognitives pour la population selon la procédure définie par Sanders (1975). Le critère d'apprentissage est l'atteinte par le sujet d'un taux d'erreurs inférieur à $5 \%$ et d'un coefficient de variation dans les performances cognitives inférieur à $15 \%$. Ensuite chaque sujet a réalisé trois sessions randomisées séparées de $48 \mathrm{~h}$ au minimum. Dans ce travail, chaque sujet réalisait les 3 sessions à la même heure de la journée pour constituer 3 groupes appariés : supplémenté avec le complexe $(n=56$, groupe $S)$, caféine seule $(n=56$, groupe $C)$, et placebo $(n=56$ groupe $P 1)$.

Le placebo, la caféine et le complexe (Isoxan Actiflash ${ }^{\circledR}$, NHS, Rungis, France) ont été présentés 
sous forme effervescente dans un verre opaque contenant environ $20 \mathrm{cl}$ d'eau. La composition du complexe vitamines et Guarana est présentée en tableau 1. La condition caféine respecte le même dosage en caféine que celui contenu dans le complexe $(60 \mathrm{mg})$. Une dose de $60 \mathrm{mg}$ de caféine a été présentée, prélevée à partir d'une solution dans laquelle un comprimé de $\mathrm{Natrol}^{\circledR}$ à $200 \mathrm{mg}$ (Natrol Caféine ${ }^{\mathbb{R}}$, Biovéa, Le Mans, France) a été dilué.

Lors de chaque session les tests cognitifs ont été présentés aux sujets pendant une période de 3 heures, immédiatement avant l'ingestion (temps $0:$ pré test), 15 minutes après l'ingestion, puis à partir de la $15^{\prime}$ minute après la prise du complément, toutes les 15 minutes pour les tâches de temps de réaction simple et la tâche décisionnelle et toutes les heures pour la tâche de vigilance. La durée maximale des tests cognitifs a été de $8,4 \pm 2$ min pour chaque présentation.

\subsection{Mesures}

\subsubsection{Test de temps de réaction simple (TRS)}

Le TRS a été mesuré, le sujet assis devant un ordinateur à une distance standardisée $(50 \mathrm{~cm})$. Le sujet tenait un déclencheur dans sa main préférentielle le pouce appuyé sur le bouton. Le TRS était défini comme le temps nécessaire au sujet pour enlever son pouce du bouton après l'apparition du stimulus. Le stimulus lumineux apparaissait au centre d'un écran et était séparé par une période préliminaire d'une durée aléatoire (3-5 s). La moyenne des TRS a été calculée sur 20 répétitions. Un temps de réaction inférieur à $160 \mathrm{~ms}$ était considéré comme une réponse anticipée (Collardeau et al., 2001).

\subsubsection{Test de décision de type Go no Go}

La tâche de Go no Go est similaire à celle décrite précédemment par Scholz et al. (2009) : les sujets devaient appuyer sur une touche en réponse à un stimulus " go » fréquent et se retenir d'appuyer sur la touche (ne pas appuyer) dans le cas d'un stimulus « no-go » rare.

\subsubsection{Mesure de la vigilance}

Le test a été présenté avant l'ingestion du complément, à la fin de la $1^{\text {re }}$ et $2^{\mathrm{e}}$ heure et à la fin de l'épreuve $(3 \mathrm{~h})$. Le test évalue la performance attentionnelle du sujet dans une tâche d'attention focalisée. Le sujet doit repérer sur une page contenant 25 signes, une forme particulière qui lui a été présentée en début de page. Le score prend en compte à la fois la vitesse d'exécution et l'exactitude des réponses (Lagarde \& Batejat, 1994).

\subsubsection{Mesure de la fréquence cardiaque et de la pression artérielle}

La fréquence cardiaque a été enregistrée à l'aire d'un cardio-fréquence mètre de type Polar RS 800 (Polar Electro Oy, Kempele, Finland). Cette mesure a été effectuée en continu pendant les 3 heures de chaque session. Les valeurs relevées la minute précédant les mesures cognitives sont utilisées pour l'analyse des résultats. La pression artérielle a été mesurée par pléthysmographie au doigt (Finapres, Ohmeda, Englewood, CO). Pour éviter tout effet hydrostatique il était demandé au sujet de garder la main équipée du capteur au niveau du coeur. Les pressions artérielles systolique (PAS) diastolique (PAD) et moyenne (PAM) ont été enregistrées.

\subsection{Analyse statistique}

Les paramètres sont décrits selon la moyenne et l'écart-type (ET). L'effet du type d'ingestion sur la performance cognitive a été étudié par une analyse de variance à deux facteurs Anova $2 \mathrm{R}$ (groupe (3 conditions) X période en mesure répétée) et par un test post-hoc de Newman-Keuls en utilisant les mesures cognitives et physiologiques comme variables dépendantes. Le seuil de signification était fixé à $P<0,05$.

\section{Résultats}




\subsection{Test de temps de réaction simple (TRS)}

\subsubsection{Temps de réponse}

$L$ 'Anova révèle un effet significatif de la période sur le temps de réaction simple $(F(8,1320)=$ $11136,2, p=0,000$, eta-deux $=0,88)$ ainsi qu'un effet d'interaction période vs. condition $(F(16,1320)$ $=8,8, p 0,000$, eta-deux $=0,90$ ). Dans toutes les conditions une dégradation de la performance (i.e. augmentation significative du temps de réaction simple) est observé avec la durée, après la 30' minute dans la condition $\mathrm{P} 1$ (pour $\mathrm{Pl}+6,2 \pm 1,4 \%, p<0,05$ ) et après 45 minutes dans les deux autres conditions (respectivement pour $\mathrm{S}, \mathrm{C}$ et $\mathrm{Pl}$ à $45 \mathrm{~min}:+11,4 \pm 1,8 \%$ vs. $+13,6 \pm 2,5 \%$ vs. $+15,8 \pm 2,1$ $\%, p<0,05$ ). La performance en TRS se stabilise ensuite (Fig. la).

\subsubsection{Erreur de réponse}

Aucun effet statistiquement significatif de la période ou de la condition n'est observé sur le pourcentage de réponses anticipées. Néanmoins, les conditions $\mathrm{S}$ et $\mathrm{C}$ se caractérisent par une grande variabilité inter individuelle et inter période partir de $45 \mathrm{~min}$ alors que le nombre de réponses anticipées est stable dans la condition Pl (Fig. lb).

\subsection{Test de décision de type Go no Go}

\subsubsection{Temps de réponse}

L'Anova révèle un effet significatif de la période sur le temps de réponse $(F(8,1320)=74388,2, p=$ $0,000$, eta-deux $=0,92)$ ainsi qu'un effet d'interaction période vs. condition $\left(F(16,1320)={ }^{8} 44{ }^{75} 4 p=\right.$ 0,000 , eta-deux $=0,93$ ). Dans les conditions $\mathrm{S}$ et $\mathrm{C}$ une amélioration de la performance (i.e. diminution du temps de réponse) est significative à partir de la 30' minute (respectivement pour les conditions $\mathrm{S}$ et C : $-3,8 \pm 0,8 \%$ vs. $-1,3 \pm 0,5 \%, p<0,05)$. Comparée au pré-test, cette amélioration de la réponse est significative dans les deux conditions jusqu'à la $120 \mathrm{e}$ minute $(p<0,05)$. A la $45 \mathrm{e}$ minute le temps de réponse est significativement plus long pour la condition placebo comparé à la valeur pré test indiquant une altération significative de la performance (différence moyenne des temps de réponse entre $\mathrm{S}$ et $\mathrm{P} 1$ à $45 \min :+7,3 \pm 1,2 \%, p<0,05)$. A partir de la 45 e minute une différence significative est également observée entre la condition $\mathrm{C}$ et la condition $\mathrm{S}$ et ceci jusqu'à la $90 \mathrm{e}$ minute. Le temps de réponse est significativement plus long dans la condition $\mathrm{C}$ comparée à la condition $\mathrm{S}$ (différence moyenne des temps de réponse entre $\mathrm{S}$ et $\mathrm{C}$ à $90 \min : 5,4 \pm 0,4 \%, p<0,05$ ). A partir de 120 minutes une nouvelle dégradation significative est observée pour la condition P1 alors que la performance reste stable pour les deux autres modalités (Fig. 2a).

\subsubsection{Erreur de réponse}

Aucun effet significatif de la période ou de la condition n'est observée sur les erreurs de réponses au test décisionnel (Fig. 2b).

\subsection{Test de vigilance}

L'Anova révèle un effet significatif de la période sur le temps de réponse $(F(3,495)=93710,6, p=$ $0,000$, eta-deux $=0,95)$ ainsi qu'un effet d'interaction période vs. condition $(F(6,495)=8814,3, p=$ 0,000 , eta-deux $=0,65$. Dans la condition placébo cette augmentation est significative à partir de la 1 " heure (respectivement pour S, C et Pl lors de la $1^{\text {re }}$ heure : $+5,7 \pm 7,6 \%$, NS vs. $+7,3 \pm 6,9 \%$, NS vs. $+38,1 \pm 12,2 \%, P<0,05)$. Dans la condition $\mathrm{C}$ et $\mathrm{S}$ cette augmentation devient significative à partir de 2 heures (respectivement pour $\mathrm{S}$, et $\mathrm{C}$ à la $2 \mathrm{e}$ heure : $+15,1 \pm 6,8 \%$, vs. $30,2 \pm 8,8 \%, P<0,05$ ). Lors de la première heure une différence significative est relevée entre les conditions $\mathrm{S}$ et $\mathrm{C}$ et la condition $\mathrm{Pl}$. Cette différence ne persiste qu'entre les conditions $\mathrm{S}$ et Pl lors de la seconde ou de la troisième heure avec une moindre dégradation de la performance dans la condition S comparée à Pl (Fig. 3a). 
Aucun effet de la période ou de la modalité n'a été observée sur le taux d'erreurs (Fig. 3b).

\section{4 Évolution de la pression artérielle et de la fréquence cardiaque de repos}

Aucune variation de la PA systolique (Fig. 4a) ou de la fréquence cardiaque (Fig. 4b) n'est observée quelles soient les conditions, au cours de la session les valeurs restent toujours dans les valeurs normales de PA systoliques ( $90140 \mathrm{~mm} \mathrm{Hg}$ ) ou de FC de repos.

\section{Discussion}

Les résultats de cette étude montrent une amélioration de la performance cognitive décisionnelle et une moindre dégradation de la vigilance avec le temps à la suite de l'ingestion du complexe comportant du Guarana et des vitamines ou de caféine sans effet négatif sur la fréquence cardiaque ou la pression artérielle. Un résultat original de cette étude est que, lorsqu'elle est comparée à une dose de caféine seule, l'ingestion du complexe vitamine et Guarana se traduit par une performance cognitive décisionnelle significativement plus élevée. En revanche aucun effet significatif n'est observé sur la performance en temps de réaction simple.

L'amélioration de la performance cognitive décisionnelle à la suite de l'ingestion de Guarana est similaire à celle observée dans les rares études précédentes comparant ingestion de Guarana et placebo (Kennedy et al., 2004; Haskell et al., 2007; Kennedy et al., 2004, 2008). Dans l'une des premières études sur ce type de complément, Kennedy et al. (2004) ont observé que l'administration de 75 mg de Guarana chez 28 sujets en bonne santé augmentait la performance dans des tâches de mémorisation. Par la suite Haskell et al. (2007) ont étudié l'effet de différentes doses de Guarana (37,5, 75, 150 and $300 \mathrm{mg}$ ) chez 26 sujets. Les résultats montrent un effet significatif à partir de $75 \mathrm{mg}$ sur des tâches de vitesse de mémorisation. L'un des résultats intéressant de ce travail est le fait qu'une dose de $75 \mathrm{mg}$ de Guarana contient un niveau de caféine $(9 \mathrm{mg})$ généralement considéré comme trop faible pour obtenir des résultats sur la performance cognitive, les auteurs suggèrent ainsi que les effets du Guarana ne sont pas liés à la caféine seule. Nos résultats, qui montrent un effet différent entre la condition caféine seule et la condition avec le complexe vitamine et Guarana confirment cette hypothèse. Dans notre travail l'amélioration de la performance dans la tâche décisionnelle de Go no Go est significativement plus importante pour le complexe que pour la condition caféine seule. Cette hypothèse peut être par ailleurs également indirectement illustrée par les résultats de Kennedy \& Scholey (2004) qui ont comparé l'effet de trois doses de caféine $(33,38$, and $46 \mathrm{mg})$ sur une batterie de tests similaire à celle utilisée dans l'étude de Kennedy et al. (2008). Ils ont montré une amélioration de la performance cognitive moins importante que celle relevée par Kennedy et al. (2008) notamment en ce qui concerne le temps de réponse laissant supposer des mécanismes différents. Par ailleurs, dans une étude récente, Campos et al. (2005) ont comparé l'influence de Guarana (25 and $50 \mathrm{mg} \cdot \mathrm{kg}^{-1}$ ) et de caféine (10 and $20 \mathrm{mg} \cdot \mathrm{kg}^{-1}$ ) sur le comportement de souris, ces auteurs ont montré dans les deux cas des effets antidépresseurs des ingestions. Néanmoins seule la caféine avait un effet inhibiteur sur l'un des agonistes de l'adénosine (cyclopentyl adenosine) permettant à ces auteurs de conclure que l'effet antidépresseur de la caféine et du Guarana reposent sur des mécanismes différents. Le Guarana contient de 4 à $8 \%$ de caféine $(25,000$ to 75,000 parties par million, ppm), aussi bien que d'autres alcaloïdes tel que la theophylline (500 to $750 \mathrm{ppm}$ ) ou la theobromine (300 to $500 \mathrm{ppm}$ ), des tannins, ou des saponines. Une hypothèse explicative de cette différence entre l'ingestion du complexe et celle de caféine pourrait être alors reliée à la présence des ces autres substances psycho-actives présentes dans le Guarana notamment les tanins ou les saponines connues pour influencer la performance cognitive (Mattei et al., 1998; Ying et al., 1994). La présence dans le complexe de vitamines pourrait également avoir un effet positif sur la performance cognitive. Chez la personne âgée ou souffrant de troubles neurologiques plusieurs vitamines et minéraux sont classiquement décrits comme essentiels pour obtenir une performance optimale et semblent avoir un effet sur le fonctionnement cérébral (Calvaresi \& Bryan, 2001 Haller, 2005). Par exemple, il a été mis en évidence chez la personne âgée, une influence des vitamines du groupe B (e.g. Durga et al., 2006), de la vitamine C et E sur la performance cognitive (e.g. Balk et al., 2007). Dans une revue de la littérature récente Huskisson et al. (2007) indiquent que les vitamines hydrosolubles des groupes $\mathrm{B}$ et $\mathrm{C}$, présentes dans le complexe de notre étude semblent les plus efficaces, néanmoins ces auteurs soulignent le fait que les résultats dans ce domaine restent encore largement spéculatifs chez le sujet sain. 
Dans notre travail aucun effet positif significatif de l'ingestion du complexe ou de caféine n'a été observé sur le temps de réaction simple (TRS). Entre 15 et 30 minutes une tendance à l'amélioration de la performance est observable pour les conditions $\mathrm{S}$ et $\mathrm{C}$, mais celle-ci n'est pas significative. Seule une différence significative est relevée à la 30 e minute entre $\mathrm{S}$ et $\mathrm{Pl}$ avec une meilleure performance pour $\mathrm{S}$ et une dégradation significative de la performance pour Pl. Ce résultat est différent de ceux rapportés dans les travaux réalisés avec ingestion de caféine qui montrent une amélioration significative du TRS sans augmentation du nombre de réponses anticipées (e.g. Attwood et al., 2010; Lieberman, 2003). Néanmoins dans ces travaux les doses de caféines sont supérieures à celles utilisées dans cette étude avec des effets positifs observés pour un dosage en caféine de 200 ou $250 \mathrm{mg}$ respectivement dans les études de Lieberman et al. (2002) et de Attwood et al. (2010). Nous pouvons donc émettre l'hypothèse selon laquelle la dose de caféine contenue dans le complexe multi vitaminé avec Guarana est trop faible pour observer une amélioration de la performance dans les tâches cognitives impliquant l'efficacité des opérations perceptives (TRS) alors qu'une amélioration est observable pour les tâches impliquant les processus décisionnels.

Enfin l'un des objectifs de ce travail était d'observer une possible perturbation de l'homéostasie cardiovasculaire décrite avec l'ingestion de caféine (Clauson et al., 2003). À la suite de l'ingestion de caféine, l'une des perturbations les plus étudiée est la réponse cardiovasculaire notamment chez le sujet hypertendu indiquant les effets négatifs de l'ingestion de caféine sur la pression artérielle (Cornelis ElSohemy, 2007). Dans notre étude aucune variation significative de la pression artérielle ou de la fréquence cardiaque n'est observée dans les conditions caféine et complexe vitaminé avec Guarana. Chez le sujet sain, si les travaux menés en laboratoire et dans des conditions de travail habituelles, indiquent une augmentation de la pression systolique même pour de faibles doses de caféine, l'effet sur la fréquence cardiaque apparait plus controversé. Par ailleurs ces effets semblent être plus faibles ou inexistants chez le sujet accoutumé au café (Etherton \& Kochar, 1993 ; Jeong Dimsdale, 1990). Ainsi les résultats de notre étude réalisée chez des sujets accoutumés à la caféine sont en accord avec ces observations et n'indiquent pas de perturbation de la réponse cardiovasculaire à la suite de l'ingestion du complexe ou de la caféine seule.

En conclusion, les résultats de cette étude supportent l'effet positif du Guarana sur la performance cognitive récemment mentionné dans la littérature (Haskell et al., 2007; Kennedy et al., 2008). Dans notre étude, cet effet positif n'est observé que les tâches décisionnelles et dans une moindre mesure sur l'altération de la vigilance avec le temps. Dans le cadre de la pratique sportive, nos résultats posent la question d'un possible effet positif de ce type de complexe lors de l'exercice physique caractérisé par une évolution différenciée avec augmentation de la sollicitation physiologique des performances décisionnelles ou perceptives (Legros et al., 1992).

\section{Bibliographie}

Attwood, A., Terry, P., \& Higgs, S. (2010) Conditioned effects of caffeine on performance in humans. Physiological Behavior, 99, 286-293.

Balk, E.M., Raman, G., Tatsioni, A., Chung, M., Lau, J., \& Rosenberg, I.H. (2007). Vitamin B6, B12 and folic acid supplementation and cognitive function. A systematic review of randomized trials. Archives of Internal Medicine, 167, 21-30.

Brisswalter, J., Collardeau, M., \& Arcelin, R. (2002). Effects of acute physical exercise characteristics on cognitive performance. Sports Medicine, 32, 555-566.

Calvaresi, E., \& Bryan, J.B. (2001). Vitamins, cognition, and aging: a review. Journal of Gerontology: Psychological Sciences, 56, 327-339.

Campos, A.R., Barros, A.I., Albuquerque, F.A., Leal, L.K., \& Rao, V.S. (2005). Acute effects of guarana (Paullinia cupana Mart.) on mouse behaviour in forced swimming and open field tests. Phytotherapy 
Cornelis, M.C., El-Sohemy, A. (2007). Coffee, caffeine, and coronary heart disease. Current Opinion in Clinical Nutrition \& Metabolic Care, 10, 745-751.

Clauson, K.A., Shields, K.M., McQueen, C.E., \& Persad, N. (2003). Safety issues associated with commercially available energy drinks. Journal of the American Pharmacists Association, 48, 55-63.

Collardeau, M., Brisswalter, J., \& Audiffren, M. (2001). Effects of a prolonged run on simple reaction time performance in well trained runners. Perceptual and Motor Skills, 93, 679-689.

Durga, J., van Boxtel, M.P., Schouten, E.G., Bots, M.L., Kok, F.J., Verhoef, P. (2006). Folate and the methylenetetrahydrofolate reductase 677C-4T mutation correlate with cognitive performance. Neurobiology of Aging, 27, 334343.

Etherton, G.M., \& Kochar, M.S. (1993) Coffee. Facts and controversies. Archives of Family Medicine, 2, 317-322.

Foskett, A., \& Gant, N. (2009) Caffeine enhances cognitive function and skill performance during simulated soccer activity. International Journal of Sport Nutrition and Exercise Metabolism, 19, 410423.

Fredholm, B.B., Bättig, K., Holmén, J., Nehlig, A., \& Zvartau, E.E. (1999) Actions of caffeine in the brain with special reference to factors that contribute to its widespread use, Pharmacological Review, 51, 83133.

Haller, J. (2005). Vitamins and brain function. In H.R. Lieberman, R.B. Kanarek \& C. Prasad (Eds.), Nutritional Neuroscience. Boca Raton, FL : CRC Press.

Haskell, C.F., Kennedy, D.O., Wesnes, K.A., Milne, A.L., \& Scholey, A.B. (2007). A double-blind, placebo-controlled, multi-dose evaluation of the acute behavioural effects of guarana in humans. Journal of Psychopharmacology, 21, 65-70.

Huskisson, E., Maggini, S., \& Ruf, M. (2007). The influence of micronutrients on cognitive function and performance. The Journal of International Medical Research, 35, 277289.

Jeong, D.U., \& Dimsdale, J.E. (1990). The effects of caffeine on blood pressure in the work environment. American Journal of Hypertension, 3, 749-753.

Johnson, D. \& Merullo, D.J. (2000). Caffeine, Bender, and sentry duty : effects of a mild stimulant on vigilance and marksmanship. In K. Friedel, H.R. Lieberman, D.H. Ryan \& G.A. Bray (Eds.), Pennington Center Nutrition Series, Volume 10: Countermeasures for Battlefield Stressors. Baton Rouge : Louisiana State University Press.

Kennedy, D.O., \& Scholey, A.B. (2004). A glucose-caffeine "Energy Drink" ameliorates subjective and performance deficits during prolonged cognitive demand. Appetite, 42, 331-333.

Kennedy, D.O., Haskell, C.F., Wesnes, K.A., \& Scholey, A.B. (2004). Improved cognitive performance in human volunteers following administration of guarana (Paullinia cupana) extract: Comparison and interaction with Panax ginseng. Pharmacology, Biochemistry and Behavior, 79, 401-411.

Kennedy, D.O., Haskell, C.F., Robertson, B., Reay, J., Brewster-Maund, C., Luedemann, J., Maggini, S., Ruf, M., Zangara, A., \& Scholey, A.B. (2008). Improved cognitive performance and mental fatigue following a multi-vitamin and mineral supplement with added guarana (Paullinia cupana). Appetite, 53, 506-513. 
Lagarde, D., \& Batejat, D. (1994). Evaluation of drowsiness during prolonged sleep deprivation. Clinical Neurophysiology, 24, 35-44.

Lambourne, K., \& Tomporowski, P. (2010). The effect of exercise-induced arousal on cognitive task performance: a meta-regression analysis. Brain Research, 23, 12-24.

Legros, P., Delignières, D., Durand, M., \& Brisswalter, J. (1992). Influence de l'effort physique sur le temps de réaction simple et de choix chez des basketteurs de haut-niveau. Science el Sports, 7, 9-14.

Lieberman, H.R. (2003). Nutrition, brain function and cognitive performance. Appetite, 40, 245-254.

Lieberman, H.R., Tharion, W.J., Shukitt-Hale, B., Speckman, K.L., \& Tulley R. (2002). Effects of caffeine, sleep loss, and stress on cognitive performance and mood during U.S. Navy SEAL training, Psychopharmacology, 164, 250-261.

Mattei, R., Dias, R.F., Espinola, E.B., Carlini, E.A., \& Barros, S.B.M. (1998). Guarana (Paullinia cupana): Toxic behavioural effects in laboratory animals and antioxidant activity in vitro. Journal of Ethnopharmacology, 60, 111-116.

McLellan, T.M., Kamimori, G.H., Voss, D.M., Tate, C., \& Smith, S.J. (2007). Caffeine effects on physical and cognitive performance during sustained operations. Aviation Space Environmental Medicine, 78, 871-877.

Rogers, N.L., \& Dinges, D.F. (2005). Caffeine: implications for alertness in athletes. Clinical and Sports Medicine, 24, 1-13.

Sanders, A.F. (1975). Structural and functional aspects of the reaction process. In S. Dornic (Ed), Attention and performance VI (pp. 3-25), New York : Wiley.

Scholz, U., La Marca, R., Nater, U.M., Aberle, I., Ehlert, U., Hornung, R., Martin, M., \& Kliegel, M. (2009). Go no-go performance under psychosocial stress: beneficial effects of implementation intentions. Neurobiology of Learning and Memory, 91, 89-92.

Ying, Y., Zang, J.T., Shi, C.Z., \& Liu, Y. (1994). Study on the nootropic mechanism of ginsenoside Rbland Rb1-influene on mouse brain development. Acta Pharmacologica Sinica, 29, 241-245. 
Tableau 1. Composition du complexe vitamine et Guarana (Isoxan, Actiflash ${ }^{\circledR}$, NHS, Rungis, France).

\begin{tabular}{|c|c|c|c|}
\hline composant & par comprimé & par jour & $\begin{array}{l}\text { ANC } 2001 \\
\text { (F) } 1800 \mathrm{kcal}^{-1} \mathrm{j}^{-1} \text { (H) } 2200 \mathrm{kcal}^{-1}{ }^{-1} \\
\text { à } \\
\text { (F) } 5800 \mathrm{kcal} . \mathrm{j}^{-1} / \text { (H) } 6200 \mathrm{kcal} . \mathrm{j}^{-1}\end{array}$ \\
\hline vitamine $\mathrm{C}$ & $180 \mathrm{mg}$ & $180 \mathrm{mg}$ & 110 à $120 \mathrm{mg}$ \\
\hline vitamine B1 & $0,705 \mathrm{mg}$ & $0,705 \mathrm{mg}$ & 1,1 à $1,3 \mathrm{mg}$ \\
\hline vitamine B2 & $0,798 \mathrm{mg}$ & $0,798 \mathrm{mg}$ & 1,5 à $1,6 \mathrm{mg}$ \\
\hline vitamine B3 & $9,005 \mathrm{mg}$ & $9,005 \mathrm{mg}$ & 11 à $14 \mathrm{mg}$ \\
\hline vitamine B6 & $1,003 \mathrm{mg}$ & $1,003 \mathrm{mg}$ & 1,5 à $2,2 \mathrm{mg}$ \\
\hline zinc & $7,490 \mathrm{mg}$ & $7,490 \mathrm{mg}$ & 9 à $12 \mathrm{mg}$ \\
\hline Guarana extraits & $300 \mathrm{mg}$ & $300 \mathrm{mg}$ & NA \\
\hline Ginseng extraits & $100 \mathrm{mg}$ & $100 \mathrm{mg}$ & NA \\
\hline
\end{tabular}


Fig. 1. Évolution du temps de réponse (1a) et du nombre de réponses anticipées (1b, erreurs en \%) dans la tâche de temps de réaction dans les trois conditions.

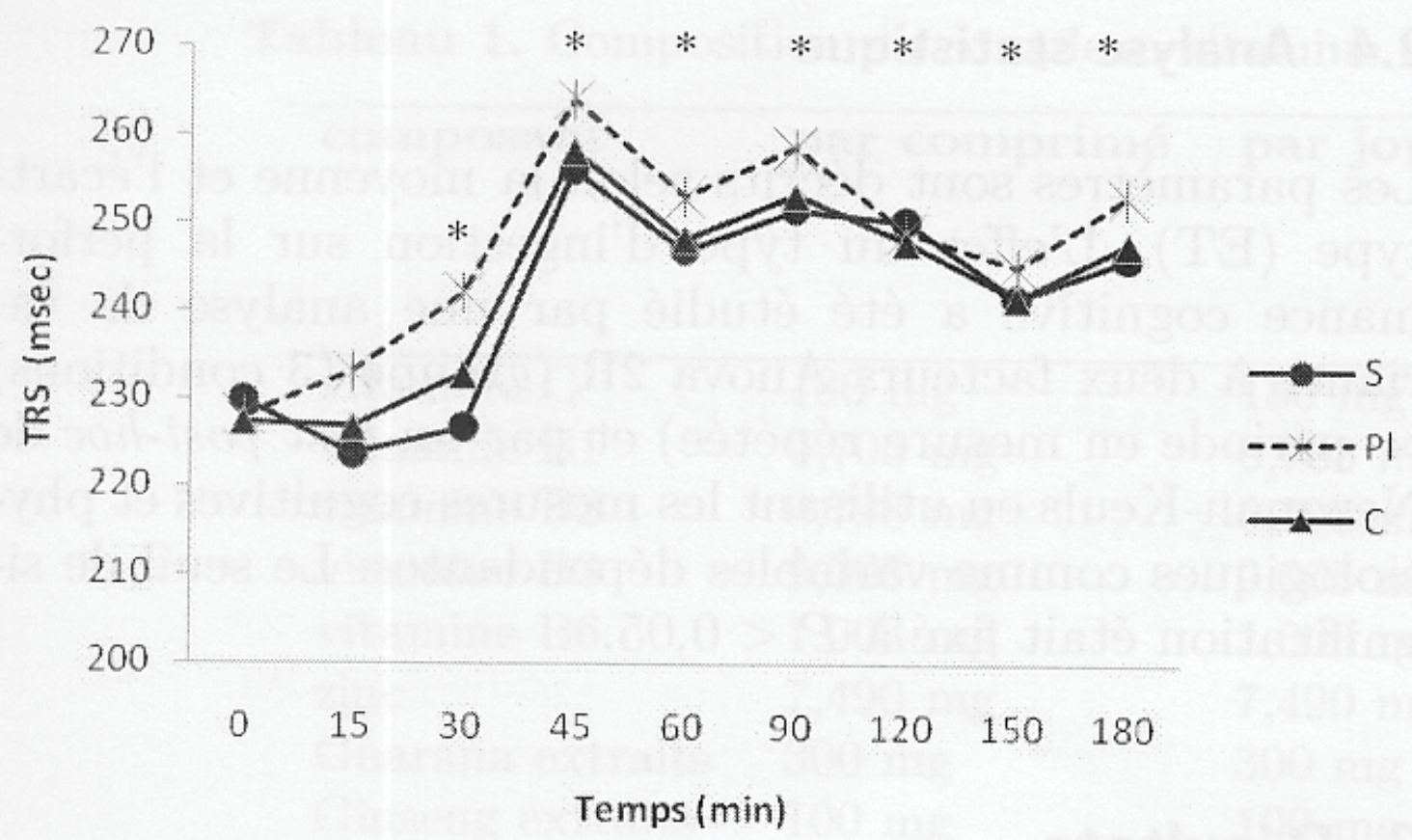

(a)

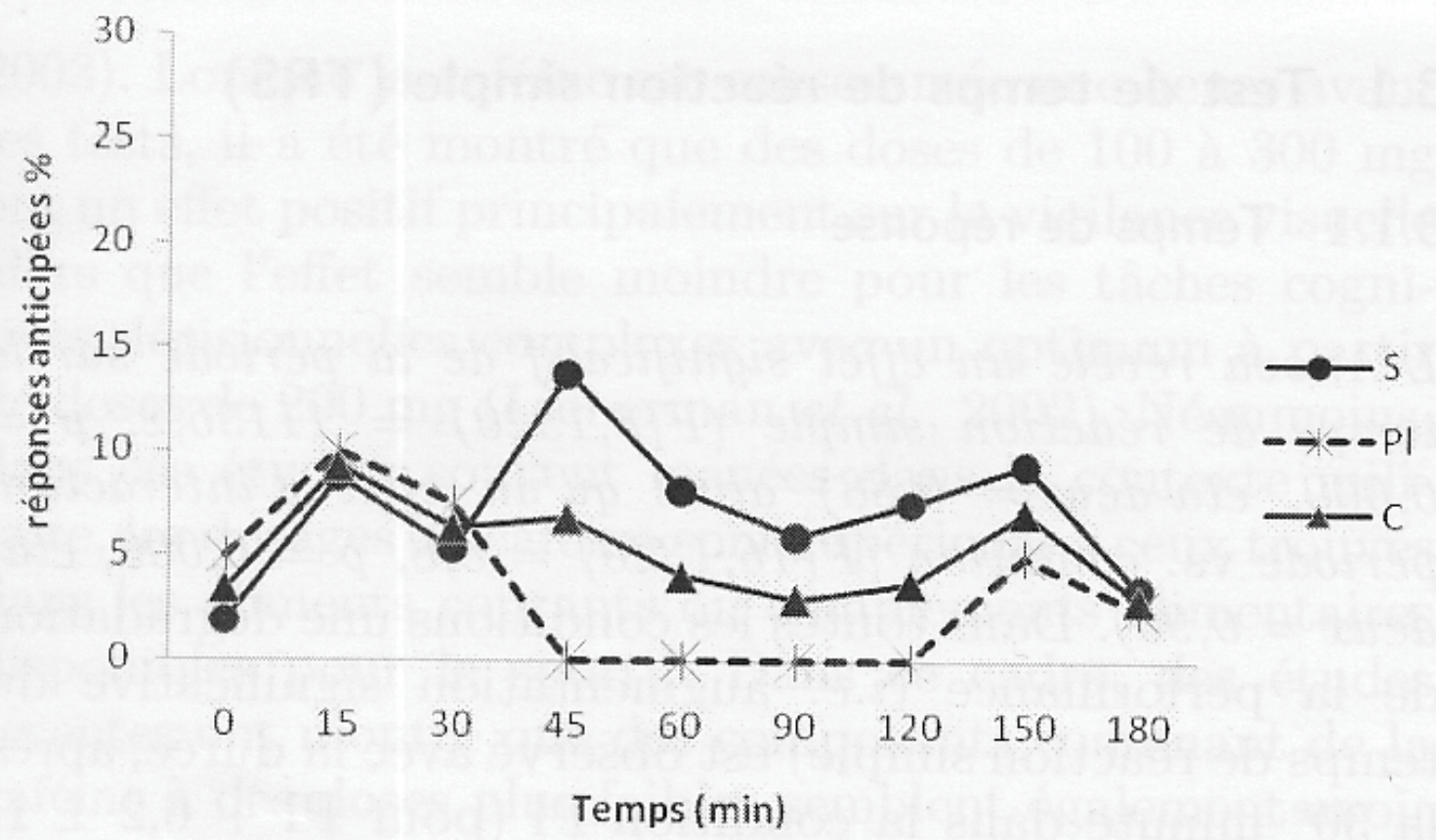

(b)

*Pour une différence significative avec le pré test $(p<0,05)$. 
Fig. 2. Évolution du temps de réponse (2a) et du nombre d'erreurs (2b) dans la tâche de Go no Go dans les trois conditions.

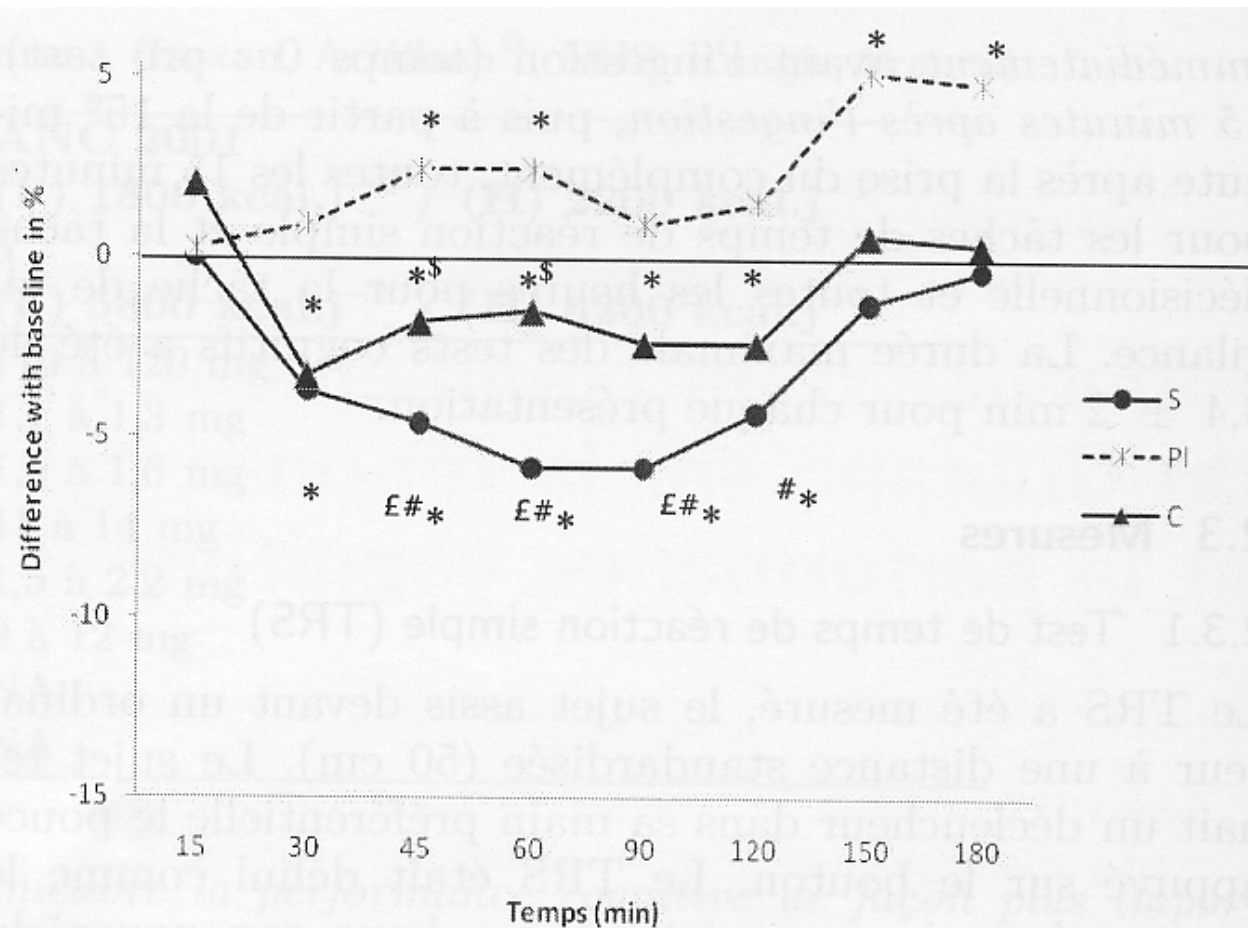

(a)

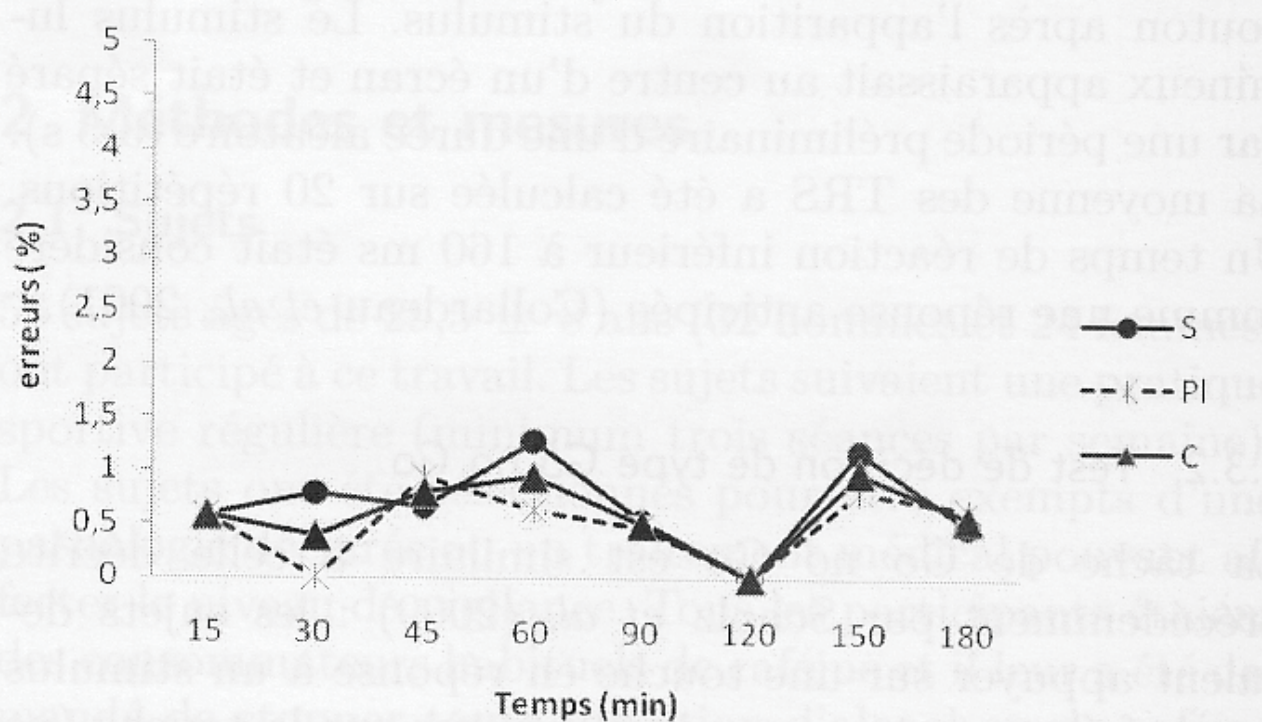

(b)

*Pour une différence significative avec le pré test $(p<0,05)$, \#pour une différence significative entre la condition S et la condition P1 $(p<0,05)$, \$ pour une différence significative entre la condition $\mathrm{C}$ et la condition $\mathrm{P} 1(p<0,05),{ }^{\text {'e }}$ pour une différence significative entre la condition $\mathrm{C}$ et la condition $\mathrm{S}(p<$ $0,05)$. 
Fig. 3. Évolution du temps de réponse (3a) et du nombre d'erreurs (3b) dans la tâche de vigilance dans les trois conditions.

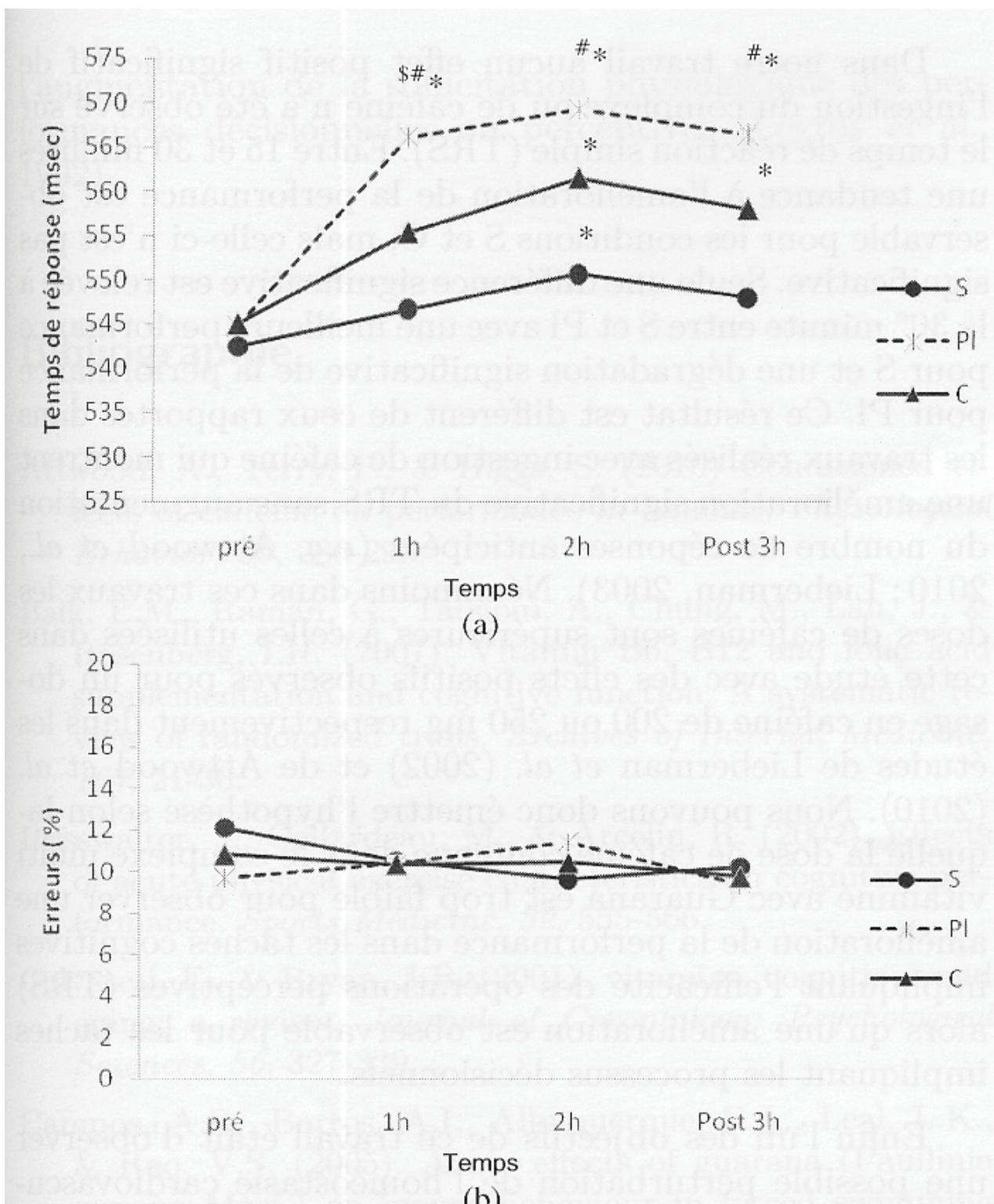

(b)

* Pour une différence significative avec le pré test $(\mathrm{p}<0,05),{ }^{\#}$ pour une différence significative entre la condition $\mathrm{S}$ et la condition $\mathrm{P} 1(p<0,05),{ }^{\$}$ pour une différence significative entre la condition $\mathrm{C}$ et la condition $\mathrm{Pl}(\mathrm{p}<0,05)$. 
Fig. 4. Évolution de la pression artérielle (4a) et de la fréquence cardiaque (4b) de repos dans les trois conditions.

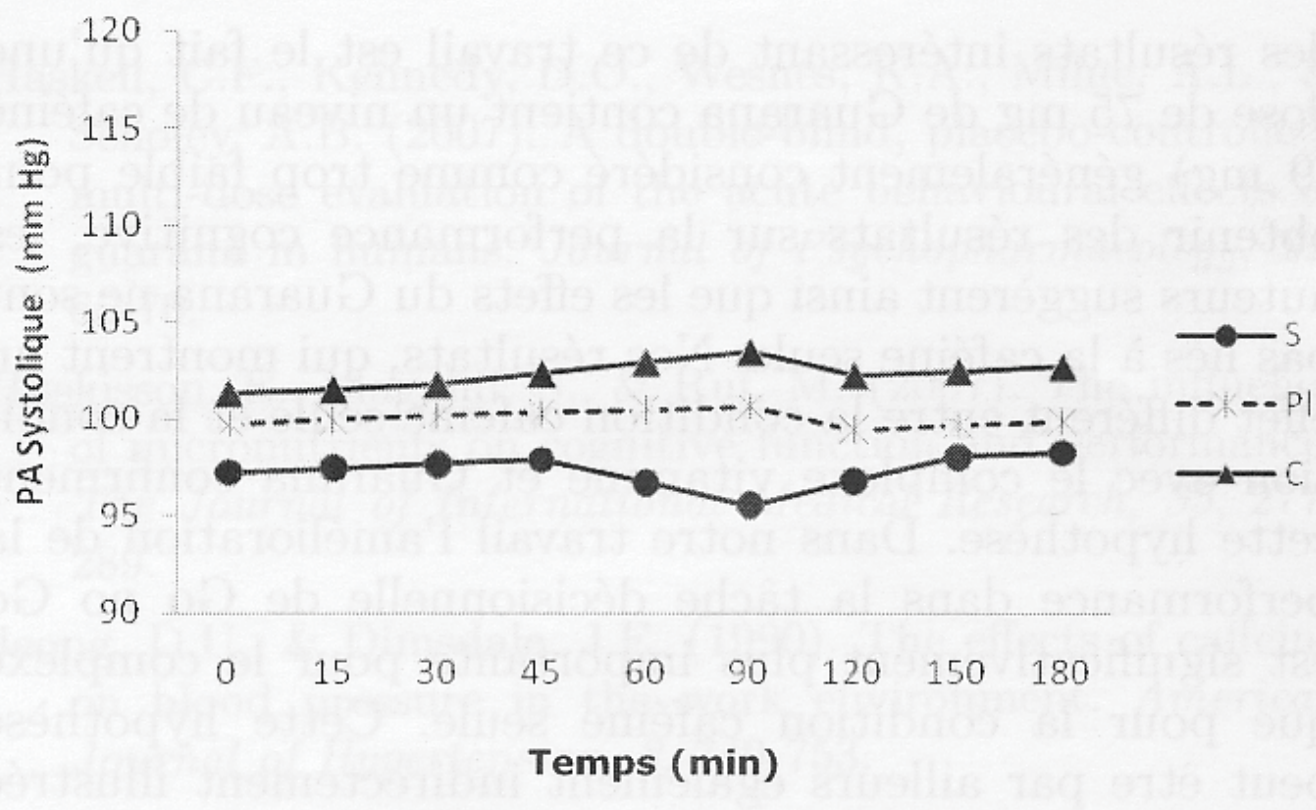

(a)

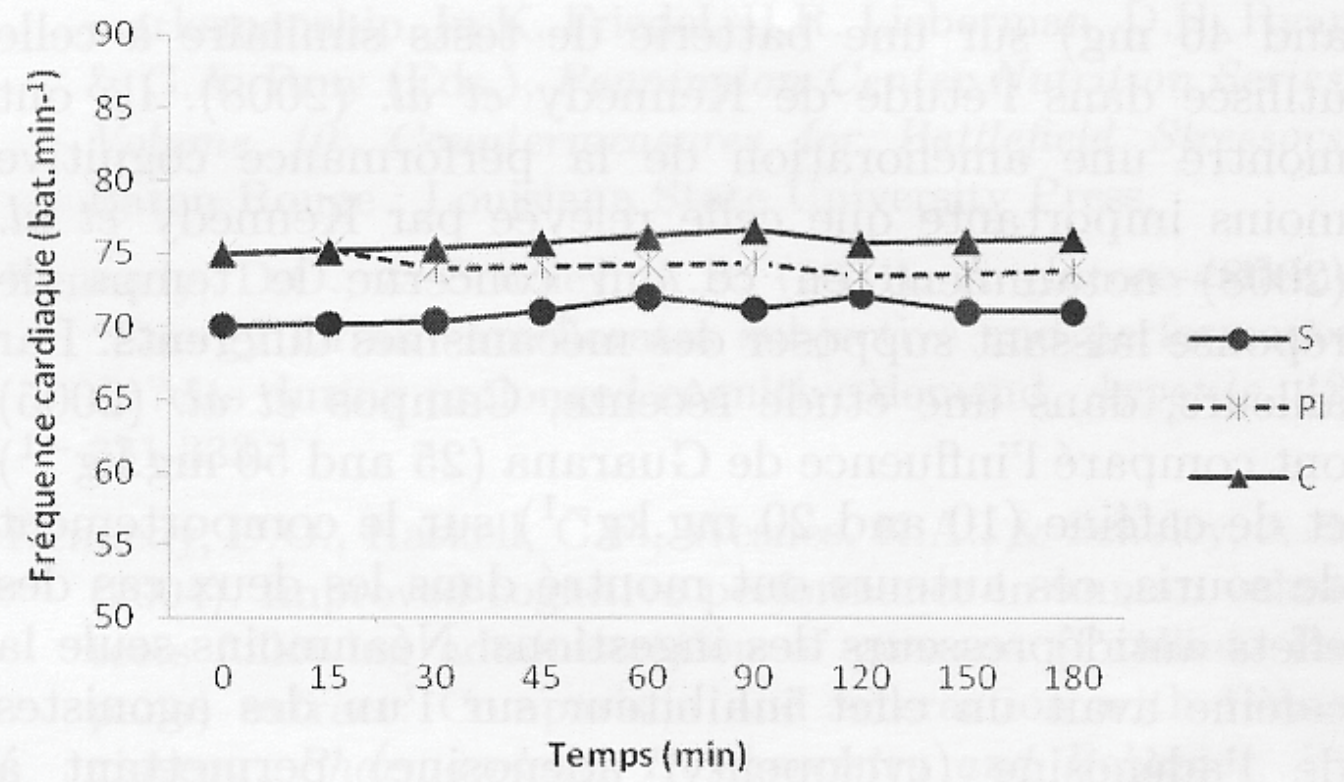

(b) 